VII.

\title{
Zur Technik der Dislocierung der Harnröhre bei der Hypospadie und anderen Defekten und Verletzungen der Harnröhre.
}

\author{
Von \\ Dr. Carl Beck, \\ Professor der Chirurgie and Chefchirurg in Nerryork.
}

(Hit 3 Abbildangen.)

Die zeitweiligen Viberfolge, welche bei der Anwendung meiner Methode der Dislocierung der Iarnröhre beobachtet werden, veranlassen mich, einige technische Punkte besonders hervorzuheben welche, wie es mir scheint, als unwesentlich angesehen sind und sich deshalb nicht genïgender Beachtung erfreuen.

Vor allem möchte ich davor warnen, die Methode da anznwenden, wo sich die Harnröhre nur unter erheblicher Spannung nach vorn verziehen läßit. Die Extensibilität dieses Organs ist zwar so bedeutend und augenseheinlich, daß man sich verwundert frägt, warum nicht schon früher Jemand auf den naheligenden Gedanken gekommen ist, diese Eigenschaft in konstruktivem Sinne zu verwerten. $\Lambda$ ber schlieblich hat auch sie ihre ganz bestimmten Grenzen und kann deshalb zum Beispiel bei der perinealen Form der Hypospadic unmöglich Erfolg versprechen. Auch bei demjenigen penilen Typus, weleher sich der Grenze des perinealen nähert, ist die Methode schon ein Wagnis zu nennen. Dies ist ganz besonders bei Erwachsenen der Fall, weil hier noch die Erektionsfrage erschwerend hinzutritt.

Man verlange also von der Harnröhre nicht gar zu viel. In praxi sind nun glücklicherweise dic Fälle, in welchen die Vorwärtsdislocierung nicht ausführbar ist, verhältnismäßig selten, da weitaus die meisten Fälle von Hypospadie balanischer oder leicht peniler Natur sind.

Die Grenzlinie zu zichen ist nicht allzuleicht, und es wird mehr 
oder minder Sache des chirurgischen Instinktes sein zu entscheiden, wann die Vorwärstdislocierung sich obne Zerrung ermöglichen läßt, oder man einen Röhrentorso aus der Harnröhrenrinne und der Skrotalhaut formt.

Wie schon angedeutet, kann man bei Kindern etwas weiter gehen als bei Erwachsenen. Dafür ist aber hinwiederum die Operation selbst etwas schwicriger auszuführen. Wer jedoch dieselbe einige male geübt hat, wird sie anch bei kleinen Kindern nicht schwieriger finden ais andere delikate plastische Eingriffe.

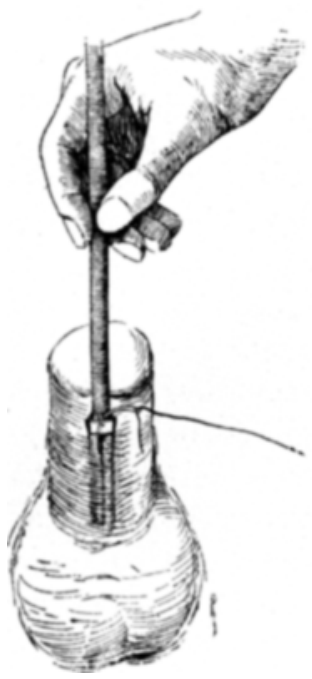

Fig. 1.

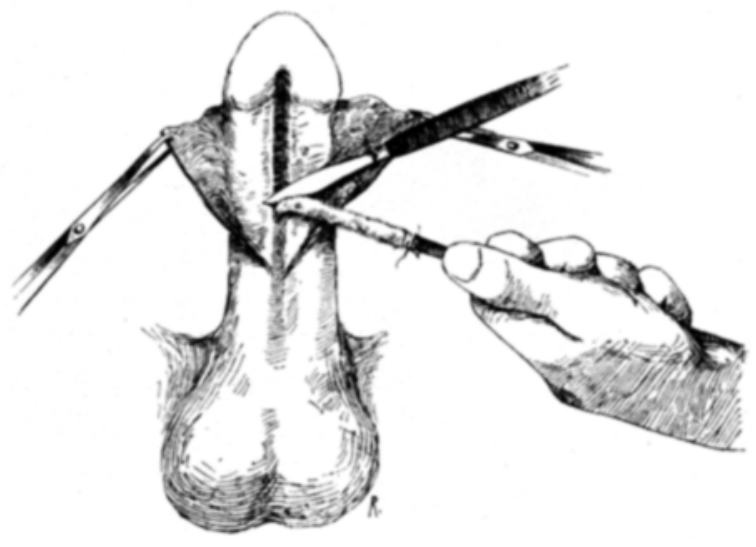

Fig. 2.

Die unterste Altersgrenze möchte ich auf den vierten Lebensnonat festsetzen. Je kleiner das Kind, desto kleiner natürlich die Harnröbre und desto schwieriger die Isolierung. Verletzungen der Harnröhre ercignen sich bei ungeübter und ungeduldiger Iland sehr leicht; glücklicherweise heilen selbst perforierte Harnröhren zumeist wieder zusammen.

Die Isolierung wird erbeblich erleichtert, wenn das Lumen der Harnröhre durch einen Gummikatheter ausgefüllt ist (vergl. meine diesbezügliche Arbeit in der Deutschen Medicinischen Wochenschrift August 1900). Benutzt man denselben gleichzeitig als Ilandhabe, indem man ihn an das Orificum befestigt, so gestaltet sich das Verfahren noch einfacher.

L'm also zu resümieren, wird die Operation damit begonnen, daß ein möglichst großer dickwandiger Gummikatheter in die Harnröhre 
eingefihrt wird. Sobald die Blase erreicht ist, wird die Katheteröffnung abgeklemmt. Nun wird (siehe Fig. 1) ein Seidenfaden mittelst einer feinen gekrümmten Nadel durch die Katheterwand und den Harnröhrenmund gefülırt. Der Faden mag, nachdem er geknotet ist, lang gelassen werden, so daf man ihn später noch zur Befestigung an die Eichelspitze verwerten kann. Bei mäßjigen Zug am Katheter (Fig. 2) läßt sich die Ilarnröhre strecken, so daß die Einschnitte sich mit größerer Sicherheit in gerader Richtung vornehmen lassen. Bei sehr kleinen Kindern muß man beim Ziehen vorsichtig sein, um die Gewebe nicht zu zerren oder gar zu. zerreißen, und empfiehlt es sich dann, die Eichelspitze mittelst einer feinen Hakenzange zu fassen und durch leichten Zug an derselben in Streckstellung zu bringen. Befestigt man eine Lage Lint oder Gaze

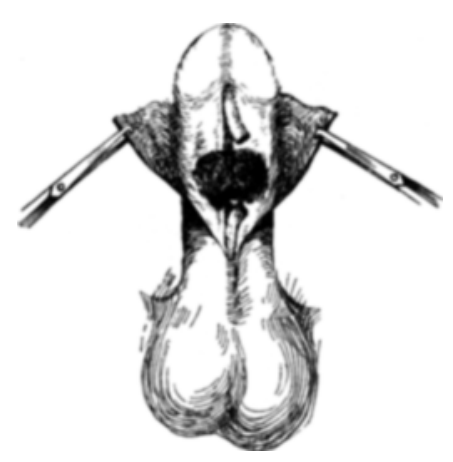

Fig. 3. auf einem Brett, so kann man die Hakenzange zugleich an derselben anhaken und durch Gegenzug so lange gestreckt halten lassen, bis die Isolierung der Harnröhre vollendet ist.

In dieser Stellung lassen sich auch die Hautlappen leichter seitlich zurückpräparieren. Bei der Formation der letzteren gehe man, namentlich wo es sich um klcine Kinder handelt, sehr vorsichtig vor und mache anfangs nur sehr oberflächliche Einschnitte.

Bei der Isolierung dient nun der Katheter nicht blob als Handhabe, sondern auch als Mentor. Man kann ihn leicht durchfühlen,' ja bei sehr jugendlichen Ilarnröhren durchschimmern seben, und so wird man bei vorsichtigem Vorgehen davor bewahrt, auf ilın einzuschneiden. Man führe die Messerzüge nicht gegen die IIarnröhre zu, sondern so zu sagen von ihr weg. und riskiere lieber, etwas Penisgewebe mit zunehmen und demnach die Blutung zu steigern, als die Harnröhre zu eröffnen.

Es ist selbstverständlich daß die Orificialportion, da sie der Kathetersutur als Halt dienen muß, so dick als möglich bleibt. Der Vorschlag von Bardenheuer und Martina, eine kleine Hautmanschette daselbst zu belassen, verdient deshalb dankenswerte Berïcksichtigung.

Wenn bei der penilen Form sich die Vorwärtsbefestigung nur unter den Zeichen von Spannung erzielen läßt, so unterstiitze ich die Naht an der Harnröhrenspitze noch dadurch, daß ich in der Nähe 
des Sulcus noch eine Eutspannungsnaht (feinstes Catgut) beiderscits. dureh die Harnröhrenwand lege.

Bei der Eichelhypospadie pflege ich den Katheter nicht länger als 24 Stunden liegen zu lassen. Bei sehr unruhigen Kindern ist das Verweilen des Katheters auf einige Tage wünschenswert. Es ist nicht absolnt notwendig, den Katheter zu beiassen. ich habe mich jedoch dureb Marwedel davon überzeugen lassen, daß ein Verweilkatheter in den meisten Fällen wenigstens für kurze Zeit vorzuziehen ist.

Eine fernere Modifikation, welche ich privater Mitteilnge des Herrn Collegen Senn verdanke, erscheint mir ebenfalls empfehlenswert. Dieselbe besteht in der Anlegung eines deltaförmigen Schnittes in der Eichel bei dem Durchstechungsmodus.

Bis jetzt habe ich nur einen einzigen Mißerfolg zu verzeichnen, welcher jedoch dem Verfahren selbst kaum zur last zu legen ist. Der cinundzwanzigjährige neuropathische Patient durehrifo am Tage nach der Operation Naht und Katheter, so dab von der Methode schließlich ganz abgesehen wurde. Ich versuehte dann, aus dem Rest der Vorbaut eine neue Harnröhre zu bilden. Zu diesem Zweck umschnitt ich die Vorhaut so weit, als sie eine Schleimhaut trug; und bog den neugebildeten I appen auf die Rinne herüber. Nachdem der Lappen dort festgewachsen war, durchschnitt ich die Brücke und begann nun aus der neu gewonnenen Unterlage eine Röhre zu formen, deren Vollendung jedoch temporär an der Cngeberdigkeit des Patienten scheiterte.

Auch ereignete es sich bei meinen Patienten wiederholt, daß einzelne der äußeren Lappennäbte nachgaben und somit ein Klaffen der Wundränder erzequgten. Es wurde dann stets eine zweite Naht darïber gelegt. Die Orificialnähte lielten jedoch immer.

Aus einer ansehnlichen Zahl von Berichten entnehme ich, dal3 meine Principien von meinen hiesigen Collegen in ausgedehntem Yaße und mit befriedigendem Erfolge geilbt werden. Es gilt dies nicht nur von der Hypospadieoperation, sondern auch ron den mannigfachen Verletzungen und Defekten der Harnröhre. In Deutschland bat neuerdings Kö ni g sich dieser Principien mit gutem Erfolg bedient. Schon am 5. August 1899 wies ich im New-York Medical Journal auf die verschiedenen Möglichkeiten der Verwendung in diesem Sinne hin. So hatte ich beispielsweise Gelegenheit, die Verziehungsmethode sogar bei der weiblichen Harnröbre zu verwenden. Bei einer alten Ilarnröhrenscheidenfistel, welche mebreren Versuchen, sie zu verschließen, getrotzt hatte, legte ich das kurze vordere Fragment frei und resecierte es. Es wurde dann die hintere Harnröhrenportion ebenfalls exponiert und nach vorwärts gezogen, so daß es an den Rest des Orificiums 
142 YII. Bkck, Zur Technik d. Dislocierung d. IIarmpöhre b. d. Iypospatie usw.

angebeftet werden konnte (sicbe New-York Medical Journal, 8. Deexmber 1900 und Deutsche Medicinische Wochenschrift 1901, No. 45.)

Bei Zerstörungen geschwüriger sowohl wie traumatischer Natur, ferner bei der Resektion alter Strikturschwielen kann das Dislocieruugsterfahren ebenfalls in Anwendung gezogen werden. So gelang es mir bei einer ganz enormen Zerstörung der Harnröhre darch einen phagedaenischen Schanker ein vollendetes Resultat zu erzielen. Es war bei dem 24 jährigen Manne nicht bloß die Eichel derart ulceriert, dab sich zwei Eichelhälften gebildet hatten, sondern das vordere Dritteil der Harnröhre war ebenfalls total zerstört worden. Nach Heilung des Cleerationsprozesses unter energischer Lokalbehandlung wurde der nekrotisierende Harnröhrenrest entfernt und der dahinter liegende gesunde IIarnröhrenteil mobilisiert und nach vorn verlegt. I)as Resultat war vollkommen. Patient wurde von mir der ,Medical Association of the Greater City of New-York" am 8. Oktober 1900 \&eheilt vorgestellt (vgl. Deutsche med. Wochenschrift 1901, Nr. 45).

Bei mehreren Fällen von ausgedehnten Verletznngen der IIarnrïhre, welche teilweise Gangrän entwickelten, erzielte ich nach Resektion des zerrissenen Harnröhrenteils völlige Heilung. Es ist überlaupt vorzuziehen, einen stark gequetsehten Harnröhrenteil zu opfern und durch ein dislociertes Ilarnröhrenstuick zu ersetzen.

Bei teilweiser Zerstörung der Harnröhre durch maligne Neubildungen habe ich im Falle eines 51 jährigen Mannes zwei 7oll reseciert und den Defekt durch beiderseitige Mobilisierung der Marnröhro gedeckt, ohne daß eine Inkurvation des Penis eintrat (Fig. 3). Denn wie ich in meinen früheren Veröffentlichungen ebenfalls schon hervorhob, läßt sich das Dislocierungsverfahren auch in retrogradem Sinne mit Frfolg anwenden; denn so gut wie man die Harnröhre nach vorn zichen kann, läßt sie sich auch nach hinten verlegen. Sclbst bej Zerstörungen durch Traumen und bei Clceration hinter der Pars praepubica gelang es mir noch, die beschädigte Harnröhrenportion zu excidieren und durch ein in der Pars pendula freigelegtes und nach rïckwärts verlagertes Stïck zu ersetzen.

Zum Schlub darf ich mir wohl gestatten, zum Frommen derjenigen Herren Collegen, welche mit der Geschichte meiner Methode nicht genuigend vertraut sind, zu erwähnen, daß ich dieselbe zum ersten Male am 17. Mai $1897 \mathrm{im}$ hiesigen St. Mark's-Hospitale ausführte und am 6. Oktober 1897 (siehe New-Yorker Medicinisebe Monatsschrift) veröffentlichte, also ein ganzes Jahr, bevor irgend welche anderweitige Veröffentlichungen über die Dislocierung der Harnröhre erschienen sind. 